\title{
A Real Time Radio Link Monitoring Using CSI ${ }^{\star}$
}

\author{
Hyukjun Oh and Jiman Hong ${ }^{\star \star}$ \\ Kwangwoon University, Seoul, Korea \\ $\{\mathrm{hj}$ _oh, gman $\} @ \mathrm{kw} . \mathrm{ac} . \mathrm{kr}$
}

\begin{abstract}
In this paper, a real time radio link monitoring scheme for wireless communication applications is proposed. It can be used as a part of the radio resource management for the efficient radio link control in wireless communication systems. The proposed method is based on the use of the channel state information transmitted or generated by mobile terminals and base stations. In contrast to the existing radio link monitoring schemes, the proposed method can provide very fast responses to the channel variations so that it is appropriate for the real time operations. In addition, the proposed scheme is computationally efficient to implement.
\end{abstract}

\section{Introduction}

Efficient utilization and allcation of the spectrum for cellular communications is certainly one of the major challenges in cellular system design [1]. In radio transmission subsystems, techniques such as deployment of time and space diversity systems, use of low noise filter and efficient equalizers, and deploy of efficient modulation schemes can be used to suppress interference and extract the desired signal [1. Co-channel interference caused by frequency reuse is the most restraining factor on the overall system capacity in wireless networks, and the main idea behind channel assignment strategies is to make use of radio propagation path loss characteristics in order to maximize the carrier-to-interference ratio (CIR) and hence increase the radio spectrum reuse efficiency. Some parameters that can be used for indicating such radio propagation channel conditions is often called channel state information. For example, signal-to-noise ratio (SNR), carrier-tointerference ratio (CIR), received signal strength indicator (RSSI), and much more.

The purpose of radio resource management (RRM) can be summarized as follows [2, 3]:

- Ensure planned coverage for each service.

- Ensure required connection quality.

- Ensure planned (low) blocking.

- Optimize the system usage in run time.

* The present research has been conducted by the Research Grant of Kwangwoon University and Seoul Metropolitan Government in 2006.

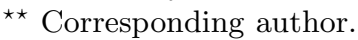


To achieve the above goals, RRM has several dedicated functions. RRM functions can be itemized to six elements: admission control, load control, packet scheduler, resource manager, handover control, and power control [3]. Any of them is very closely tied with the radio link status or condition [3]-[5]. Especially, connection based RRM functions are critically dependent on the radio link condition. Therefore, the radio link monitoring functionality is one of the most critical elements in the wireless communication system combined with RRM. In addition, the response time of radio link monitoring function determines the performance of overall RRM operations. The real time operationality of the radio link monitor or even faster response time in the real time radio link monitoring function is the key design factor.

The overall performance of RRM is much more improved as RRM response time is shortened further. As mentioned above, the basic foundation for good RRM is knowledge about the current propagation channel condition because the best RRM is supposed to react to the time-varying propagation channel condition most properly. RRM gets the required propagation channel information from the radio link monitoring function. This leads to emphasizing of the radio link monitoring functionality and the real time operation of the radio link monitoring with very fast response time is critical to improve the overall system performance. Recently, it has been noted that the real time radio link monitoring functionality plays an important role in several radio resource management related works in real time wireless communication systems recently for this reason [5], 6].

Existing radio link monitoring schemes are mostly dealt by higher layers 3][5]. In results, the response time for radio link status change of previous radio link monitoring schemes are slow and they are appropriate for non-real time or limited real time RRM functions. Hence, there is a big room for possible performance improvement of the overall system by adding the fast real-time radio link monitor. Existing previous schemes are utilizing a long-term observation of the propagation channel condition and they take reactions to the cases where there is a noticeable big change in the propagation channel condition such as radio link failure [3]-[5]. In other words, they cannot react to the current instantaneous channel condition properly. It is possible to extend the current existing schemes to deal with the instantaneous radio link state, but it is quit complex and it is even not implementable in some real-time communication systems.

In this paper, a real time radio link monitoring scheme for wireless communication applications is proposed. It can be used as a part of the radio resource management for efficient radio link control in wireless communication systems. The proposed method is based on the use of the channel state information transmitted or generated by mobile terminals and base stations. In contrast to the existing radio link monitoring schemes, the proposed method can provide very fast responses so that it is appropriate for the real time operations. In addition, the proposed scheme is computationally efficient to implement and it is appropriate for the real time operations. The proposed scheme can provide very 
efficient real time radio link monitoring functionality in the wireless communication systems combined with the radio resource management.

This paper is structured as follows: Section 2 describes our system model with the applications of wireless communications used for our work. Section 3 addresses the proposed radio link monitoring algorithm appropriate for the fast real-time wireless communications using the channel state information.

\section{System Model}

In this section, the target system model is addressed. A usual RRM system with radio link monitoring function is shown in Fig. 1 [3], which comprises several system parameters that can be used or controlled by joint combination of RRM and radio link monitor. The input of the real-time RRM system consists of two tables and a set of traffic parameters such as the number of carriers, the number of data users, the multi-slot classes, and the traffic loads of voice and data. All of these are controlled by RRM given radio link monitor results. The control outputs of the system are the radio resource related parameters such a delay calculated as Time to First Bit (TTFB), average throughput, bandwidth utilization, number of retransmissions, modulation and coding schemes, and resource allocations [3].

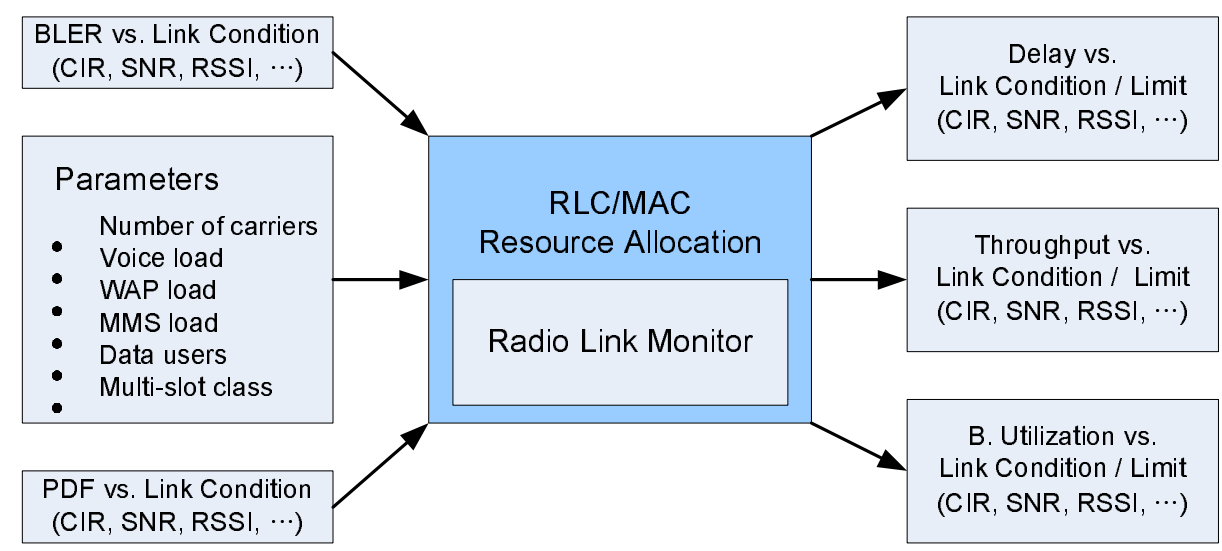

Fig. 1. A system model

So far, such important system tables have been built based on non-real time radio link monitor results obtained through long-term observable radio link parameters. It causes the performance degradation when the radio link is fast time-variant because it can reflect only long-term variations of the propagation channel. During the short-term variation of the radio link, the existing radio link scheme cannot update its radio link state fast enough because it doesn't have detailed state of the radio link or it doesn't support real-time processing [3]-[5]. In results, the design of real-time radio link monitor that is able to detect fast 
time-varying channel condition is indispensable. The importance of such a radio link monitoring capability gets more important as wireless communication systems gets more evolved to support very high speed mobile over $250 \mathrm{~km} / \mathrm{h}$.

Another thing that we must point out is what parameters or what indicators are available and appropriate for this purpose of the real-time radio link monitoring that can reflect fast varying radio link conditions. As mentioned above, several parameters are being utilized by RRM and radio link monitor. None of them is appropriate for the short-term radio link state monitoring. Most wireless communication systems have the parameter of channel state information (CSI) for the reliable transmissions. CSI's are available in the form of the signals from the base stations or in the form of direct estimations by the end-user equipment. In general, CSI is used in physical layer to react to the fast-varying propagation channel condition. It is quite complex in the sense of multiple detailed levels that it is represented. Many variants or forms of system parameters can be regarded as CSI. Any form of CSI's can be used for this purpose. The most promising and preferable one is instantaneous or short-term signal to interference ratio (SIR) or some other indicators directly related to the instantaneous SIR values. In this paper, we consider the use of one candidate of the TPC bit that is directly generated from short-term SIR values. The details are addressed in Section 3.

The overall RRM function combined with radio link monitor provide several RRM functionalities as shown in Fig. 2. It shows an example of function-level description of targeted system model [3. As shown in the figure, the radio link monitoring scheme plays a fundamental basic role in the system. Each block belongs to one of two categories of network based and connection based functions [3]:

- Network based functions

- Admission control (AC).

- Load control (LC).

- Packet scheduler (PS).

- Resource Manager (RM).

- Connection based functions.

- Handover Control (HC).

- Power Control (PC).

Because our interest is focused on the system with the application of wireless communications, the best target system would be cellular phone. Mainly, it consists of three parts: application related components like several multimedia peripherals and application processors, modem related components including baseband processors, and RF chains [6]. It is assumed that the dedicated application or modem processor is equipped with an operating system and it serves as a brain for performing the proposed radio link scheme in this paper. A real time operating system (RTOS) would be appropriate for this purpose, but we do not limit the form of possible operating system in our study, because the operating system is not of our interest in this paper. It simply provides a room for central control of the RRM and radio link monitoring scheme. The embedded 


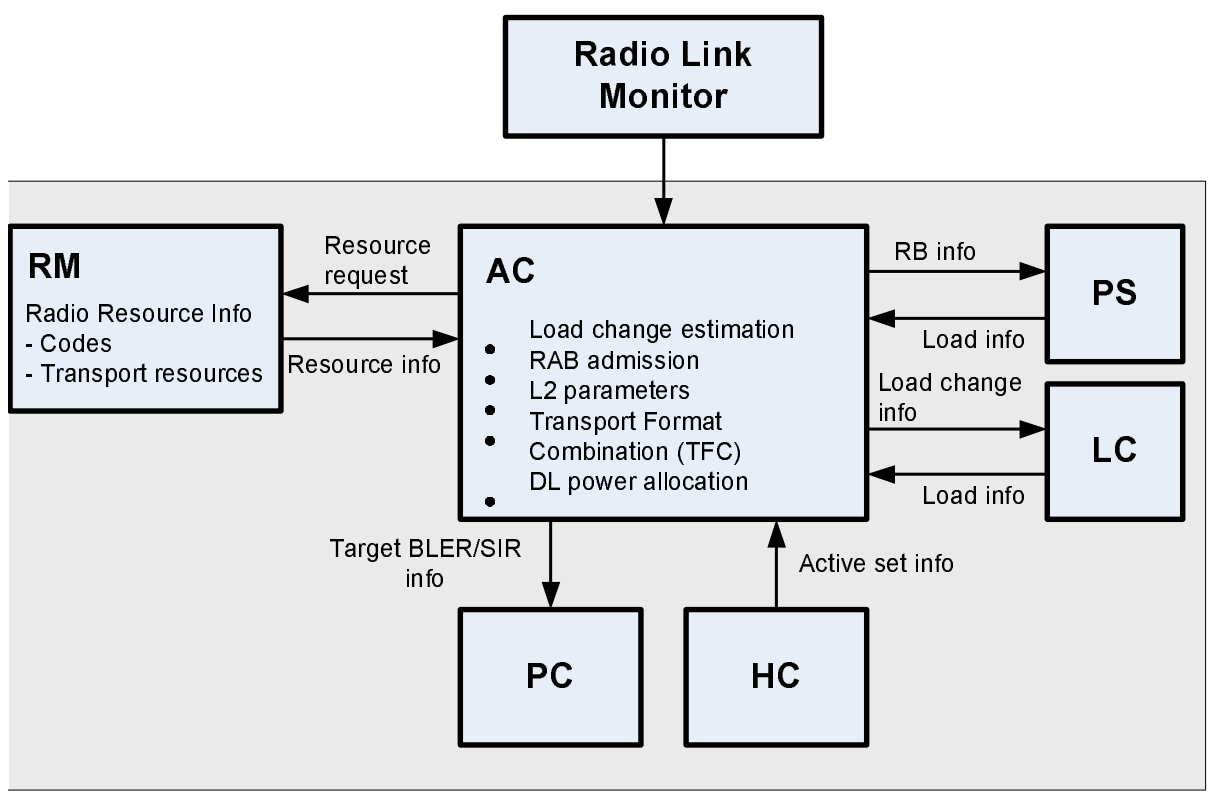

Fig. 2. An example of function-level description of the overall system

processors currently used in designs take two possible shapes: microprocessors or digital signal processors (DSP's). They can be used separately as a single component, or they can be incorporated in a larger silicon chip in the form of embedded cores along with program/data memory and other dedicated logics. In our system model, any form and any kind of dedicated processors are allowed. The proposed scheme is simple enough to support real time processing even with SW.

\section{Proposed Real Time Computationally Efficient Radio Link Monitoring Scheme}

A computationally efficient signal processing algorithm is proposed to monitor the propagation radio link condition. The algorithm should be simple to implement so that it can provide the fast response in the real-time processing and it does not add another noticeable power dissipation to the overall system. The proposed algorithm is based on CSI that is available in most wireless communication systems for the reliable transmissions [6]. In this section, we consider the CDMA and OFDM cellular systems that are the most widely accepted third generation standards for the cellular communications in the world. However, the proposed scheme is not limited to those systems. It can be generalized easily to other systems.

In CDMA systems, several different CSI's are estimated or transmitted by the end-user terminal. Some of them are mandatory and some of them are for 
performance improvements. It is natural to design the detection algorithm using mandatory CSI's. In CDMA systems, the CSI's of the transmitted power control (TPC) bits are available in plenty of time. They have binary channel state information. One is indicating that the current channel quality is good enough to satisfy the required quality of service. The other is representing the channel condition is not good enough to achieve the reliable transmissions.

The proposed radio link monitoring scheme simply estimates the frequency of occurrence of this unsatisfactory and satisfactory channel state by counting the number of the second state of the TPC bits during the given time period like one given in [6]. Then, the current channel condition is regarded as the extremely good (bad) state if the majority of the TPC bits are the first (second) state in the given time frame. That is, if the counted number of the occurrences of the corresponding CSI in the fixed time duration is larger than a threshold, the radio link monitor regards the current channel condition as the corresponding state. It can be processed in real time because of its simplicity. The proposed signal processing algorithm can be implemented in either logics or codes easily.

In OFDM systems that are hot research topics in recent activities, most popular CSI's are sub-carrier SIR's. Both of long-term and short-term SIR measurements are possible. The short-term SIR values are good real time indicators for the instantaneous radio link quality. However, it is a burden work to process short-term sub-carrier SIR values with faster response because they are quiet large amount of information to process in real-time when the number of subcarriers is large. A complex dedicated hardware would be required to process them in real time for radio link monitoring.

In this paper, we propose a novel scheme to utilize such burden CSI's efficiently for radio link monitoring. The proposed method is very simple to implement and make use of them possible for real-time processing even with SW codes.

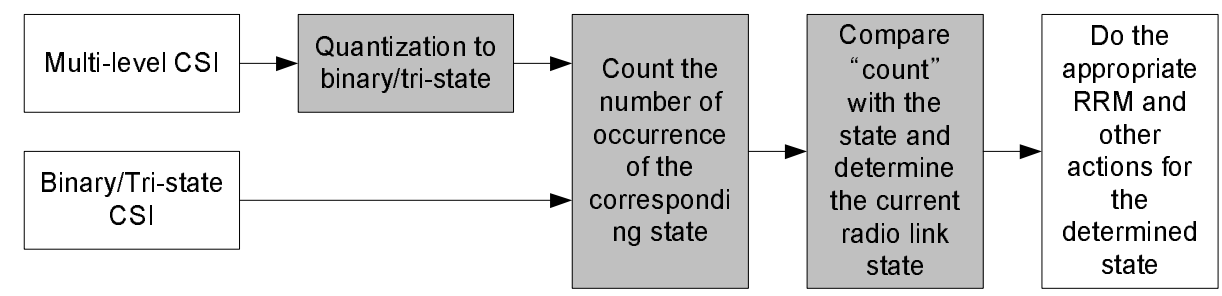

Fig. 3. The proposed real time radio link monitoring method including the quantization

The proposed method is to quantize such burden CSI information to binary or tri-states. In fact, one can try even larger number of states but it make the radio link monitoring system fail to meet our purpose of real time processing with simple codes due to the increased complexity. The proposed real time radio link monitoring scheme based on the quantization is described in Fig. 3 and 4. In any case, it can be processed in real time with fast response indicators of the 
radio link monitor. It requires only a couple of counters and a simple quantizer to implement. In Fig. 4, an example of tri-state based radio link monitoring is given.

Using our proposed method, any form of CSI's can be utilized for achieving the goal of the desirable radio link monitoring. For instances, ACK/NACK, several event-indicating flags, CRC check flag, hybrid ARQ state information, and TPC bits are appropriate for the binary state based radio link monitoring without quantization. On the other hand, SIR, RSSI, CIR, noise variance, estimated channel responses, and energy metric of decoders are useful for the real-time radio link monitoring with quantization into binary or tri-state information.

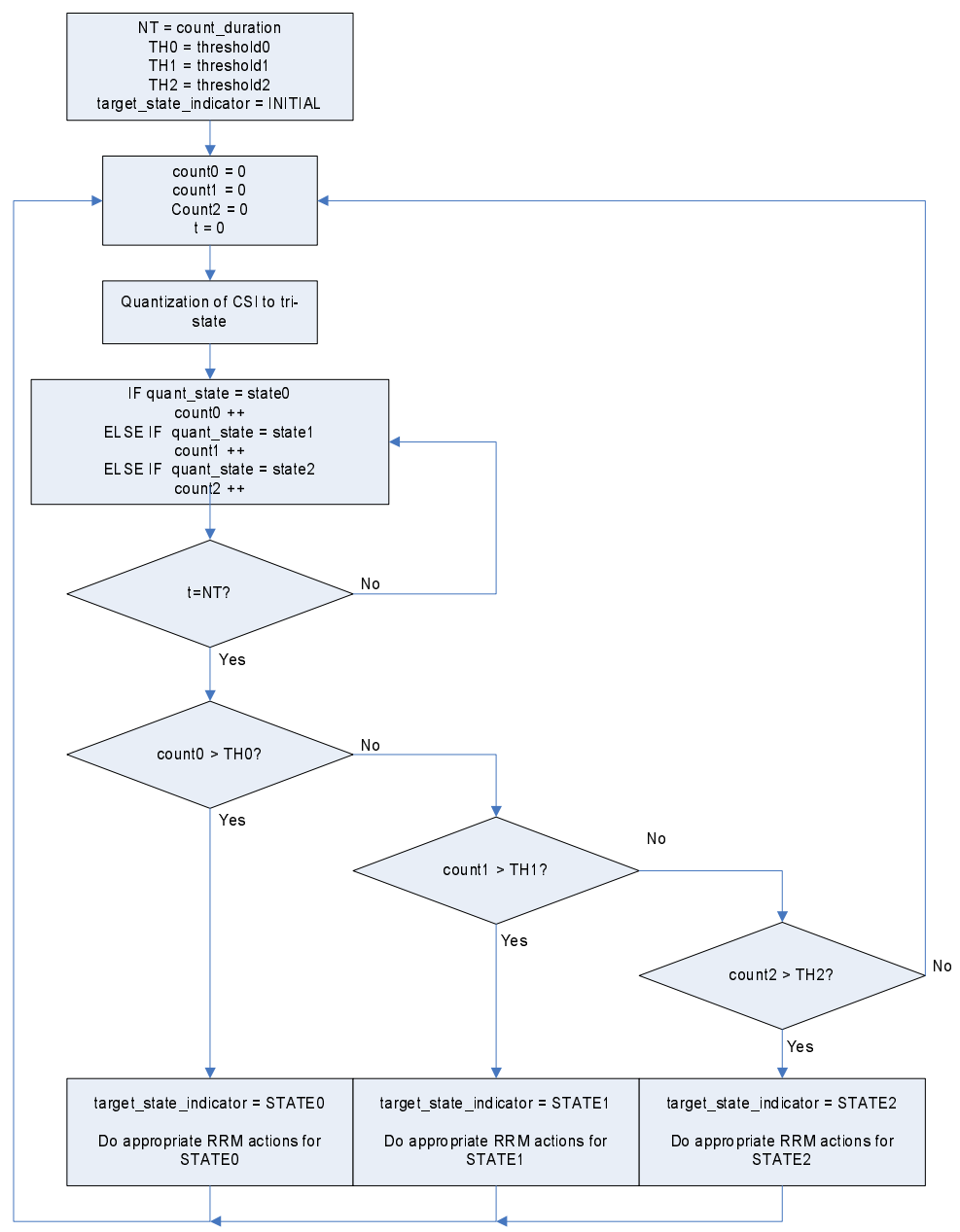

Fig. 4. An example of the proposed radio link monitoring scheme based on tri-state radio link condition 


\section{Conclusions}

In this paper, a possible way to improve the performance of the radio resource management using the real-time radio link monitoring function based on the channel state information available in usual wireless communication system has been proposed. The proposed scheme is very simple to implement and to monitor the propagation channel condition effectively so that it can provide the real-time operation even with SW codes. A signal processing algorithm to monitor the radio link channel condition is also proposed using the available channel state information combined with the quantization methodology.

\section{References}

1. Foschini, G., Golden, G., Valenzuela, R., Wolniansky, P.: Simplified Processing for High Spectral Efficiency Wireless Communication Employing Multi-Element Arrays IEEE Journals on Selected Areas in Communications, Vol. 17 (1999) 1843-1852

2. Merrill, W., Newberg, F., Sohrabi, K., Kaiser, W., Pottie, G.: Collaborative networking requirements for unattended ground sensor systems. Proc. IEEE Aerospace Conference (2003) 1-13

3. Nowicki, E.: Resource allocation for multimedia messaging services over EGPRS. Master of Science thesis, Dublin City University (2003)

4. Hattori, T., Sasaki, A., Momma, K.: A new mobile communication system using autonomous radio link control with decentralized base stations. Proc. IEEE Vehicular Technology Conference (1987) 579-586

5. Nordstrand, I., Bodin, S.: Radio link failure. US Patent No. 5487071 (1996)

6. Oh, H., Hong, J., Ahn, H.: An intelligent power management scheme for wireless embedded systems using channel state feedbacks Proc. International Conference on Fuzzy Systems and Knowledge Discovery (2005) 1170-1173 